\title{
A New Era in the Management of the Hepatitis C
}

\author{
Alfredo Arredondo Bruce, Osmani Risco Morales \\ "Amalia Simoni" Teaching Hospital, Camaguey, Cuba \\ Email: alfredoab@finlay.cmw.sld.cu, orisco@finlay.cmw.sld.cu
}

Received 26 March 2014; revised 24 April 2014; accepted 15 May 2014

Copyright (C) 2014 by authors and Scientific Research Publishing Inc.

This work is licensed under the Creative Commons Attribution International License (CC BY). http://creativecommons.org/licenses/by/4.0/

\section{(c) (i) Open Access}

\section{Abstract}

Hepatitis $\mathrm{C}$ is an infection caused by the hepatitis $\mathrm{C}$ virus that attacks the liver and leads to inflammation. The current standard-of-care regimens include a protease inhibitor-telaprevir or boceprevir-in combination with pegylated interferon and ribavirin. Hepatitis $\mathrm{C}$ treatment options on the horizon hold promise for better viral clearance with less toxicity than current regimens. There are new data about new drugs, both direct-acting antivirals while minimizing intolerable side effects or adverse events. Developed new data from 4 phase 3 trials with the hepatitis $\mathrm{C}$ drug sofosbuvir and ribavirin show that a 12-week regimen is effective in treating HCV genotypes 1 through 6. In the Annual Scientific Meeting and Postgraduate Course of the American College of Gastroenterology, different research was presented that was drawn from 4 phase 3 studies: NEUTRINO, FISSION, POSITRON and FUSION which enrolled different types of patients, who received Sofosbuvir with Peginterferon Alfa 2a and Ribavirin for 12 or 24 weeks in treatment; for all studies, the primary end point was sustained virologic response at 12 and 24 weeks posttreatment. In all studies, sofosbuvir was well tolerated, with a low incidence of adverse events. In conjunction with the suggested brief duration of this regimen, this indicates that drug combinations should improve treatment adherence compared with IFN-based treatment. In conclusion, 2 novel directacting antiviral agents_-sofosbuvir and simeprevir_target various components of the HCV genome. Advantages of these drugs include a high barrier to viral resistance, a shorter duration of treatment, once-daily dosing, absence of food restrictions, few clinically significant drug interactions, and similar efficacy in all genotypes.

\section{Keywords}

Hepatitis C, Sustained Virologic Response, Intolerable Side Effects or Adverse Events, Sofosbuvir and Simeprevir 


\section{Introduction}

Hepatitis C is an infection caused by the hepatitis C virus (HCV) that attacks the liver and leads to inflammation. The World Health Organization (WHO) estimates that about 3\% of the world's population has been infected with HCV and that there are more than 170 million chronic carriers who are at risk of developing liver cirrhosis and/or liver cancer [1].

In 2011, the American Association for the Study of Liver Diseases (AASLD) issued an updated version of its practice guidelines for the treatment of chronic genotype 1 hepatitis $\mathrm{C}$ virus (HCV) infection [2]. The current standard-of-care regimens include a protease inhibitor-telaprevir or boceprevir-in combination with pegylated interferon (PEG-IFN) and ribavirin. Protease inhibitor-based strategies for patients with genotype 1 HCV have led to high rates of sustained virologic response (SVR); however, there are several recurring concerns [2].

The disadvantages of IFN treatment are well known. Moreover, this strategy presents a complex and prolonged therapeutic course (24 - 48 weeks), low tolerability, and a low barrier to resistance, and reduced efficacy in prior null responders or cirrhotic patients. These regimens are not an option for many patients because of contraindications or intolerability to IFN [3]-[5].

Hepatitis $\mathrm{C}$ treatment options on the horizon hold promise for better viral clearance with less toxicity than current regimens. There are new data about new drugs, both direct-acting antivirals and for some investigational agents used in combination regimens for patients with genotypes 1 to 4 hepatitis C. Several trials suggest the potential for interferon- and ribavirin-free all-oral regimens for genotypes 2 and 3 hepatitis $\mathrm{C}$, and there is a variety of new combinations for the hard-to-treat genotype 1 [6].

To address these concerns, there has been a massive effort to create the ideal agent or strategy for use in updated therapeutic efforts. The pace of discovery has been unprecedented, and several agents are in the later phases of development. The approach has discovered drugs that directly target various aspects of the HCV life cycle-hopefully leading to combinations of agents that will more effectively treat patients, while minimizing intolerable side effects or adverse events.

\section{Developed}

Patients with acute hepatitis C virus (HCV) infection appear to have an excellent chance of responding to 6 months of standard therapy with interferon (IFN). Because spontaneous resolution is common, no definitive timing of therapy initiation can be recommended; however, waiting 2 - 4 months after the onset of illness seems reasonable.

Treatment of chronic HCV infection has 2 goals. The first is to achieve sustained eradication of HCV (i.e., sustained virologic response [SVR]), which is defined as the persistent absence of HCV RNA in serum 6 months or more after completing antiviral treatment. The second goal is to prevent progression to cirrhosis, hepatocellular carcinoma (HCC), and decompensated liver disease requiring liver transplantation [7].

An open-label phase 3 study conducted in Japan evaluated -oral interferon- and ribavirin-free triple combination of the investigational NS5A replication inhibitor daclatasvir, the NS3 protease inhibitor asunaprevir, and the non-nucleoside NS5B polymerase inhibitor BMS-791325. The regimen was compared in 135 interferon-ineligible or -intolerant patients with genotype $1 \mathrm{~b}$ hepatitis $\mathrm{C}$ and 87 nonresponders to interferon and ribavirin. Sustained viral response at 24 weeks was better in the ineligible or intolerant group than in the nonresponders (87.4\% vs $80.5 \%$ ). Serious adverse events occurred in $5.9 \%$ of patients, and $5.0 \%$ discontinued treatment because of adverse events - $90 \%$ of which were related to liver enzyme elevation [8].

A 12-week phase $2 \mathrm{~b}$ study evaluated the same triple combination in 166 treatment-naïve patients with genotype 1 hepatitis C. Sustained viral response at 12 weeks, considered to be a cure, was achieved in more than $90 \%$ of the 15 patients with genotype $1 \mathrm{a}$ or $1 \mathrm{~b}$ and in the 151 with or without cirrhosis [8].

New data from 4 phase 3 trials with the hepatitis C (HCV) drug sofosbuvir (SOF) and ribavirin (RBV) show that a 12-week regimen is effective in treating HCV genotypes 1 through 6. Twenty-four-week sustained virologic response (SVR) is essentially identical to 12-week SVR, bolstering confidence that the drug combination represents a cure. Those with genotype 3 infections are better served with a 16-week course of treatment [9].

The new work extends the results of the studies out to 24 weeks after treatment cessation. Twenty-four weeks was the traditional milestone for HCV treatments, but in recent years, the US Food and Drug Administration and industry have gravitated toward the 12-week time point. However, with new drugs set to greatly affect HCV treatment, it is important to consider this older benchmark [9]. 
In the Annual Scientific Meeting and Postgraduate Course of the American College of Gastroenterology, presented in San Diego, California, at October 15, 2013, was presented different research that drew from 4 phase 3 studies: Sofosbuvir With Peginterferon Alfa 2a and Ribavirin for 12 Weeks in Treatment-Naive Subjects with Chronic Genotype 1, 4, 5, or 6 HCV Infection (NEUTRINO), which enrolled treatment-naive patients with genotype (GT) 1, 4, 5, and 6 infection, each of whom received 12 weeks of SOF, peg-interferon (PEG), and ribavirin (RBV); Phase 3 Study of Sofosbuvir and Ribavirin (FISSION), which enrolled treatment-naive GT 2/3 patients to receive either 12 weeks of SOF + RBV or 24 weeks of PEG + RBV; GS-7977 + Ribavirin for 12 weeks in Subjects with Chronic Genotype 2 or 3 HCV Infection Who Are Interferon Intolerant, Interferon Ineligible or Unwilling to Take Interferon (POSITRON), which enrolled GT 2/3 patients unable or unwilling to receive interferon, who were randomly assigned to receive 12 weeks of SOF + RBV or placebo; and Sofosbuvir + Ribavirin for 12 or 16 weeks in Treatment Experienced Subjects with Chronic Genotype 2 or 3 HCV Infection (FUSION), which enrolled treatment-experienced GT2/3 patients who received 12 or 16 weeks of SOF + RBV. [10]

For all studies, the primary end point was sustained virologic response (HCV RNA $<25 \mathrm{IU} / \mathrm{mL}$ ) at 12 weeks posttreatment (SVR12) (Table 1).

Compensated cirrhosis at baseline was found in $17 \%$ of patients in the NEUTRINO study, $21 \%$ in FISSION, $18 \%$ in POSITRON, and 33\% in FUSION.

In all studies, SVR12 was higher in patients without cirrhosis. Patients with GT 2 experienced higher SVR12 rates than those with GT 3.

SVR24 rates were similar to SVR12 rates (Table 2).

Table 1. 12-week SVR rates.

\begin{tabular}{|c|c|c|c|c|c|c|c|}
\hline & \multirow{3}{*}{$\begin{array}{c}\text { GT 1, 4, 5, } 6 \\
\text { NEUTRINO } \\
\begin{array}{c}\text { SOF/PEG/RBV } \\
(n=327)\end{array}\end{array}$} & \multicolumn{6}{|c|}{ GT 2 and 3} \\
\hline & & \multicolumn{2}{|c|}{ FISSION } & \multicolumn{2}{|c|}{ POSITRON } & \multicolumn{2}{|c|}{ FUSION } \\
\hline & & $\begin{array}{c}\text { SOF/RBV } \\
(n=253)\end{array}$ & $\begin{array}{c}\text { PEG/RBV } \\
(n=243)\end{array}$ & $\begin{array}{c}\text { SOF/RBV } \\
(n=207)\end{array}$ & $\begin{array}{l}\text { Placebo } \\
(n=71)\end{array}$ & $\begin{array}{l}\text { SOF/RBV } 12 \text { week } \\
\quad(n=100)\end{array}$ & $\begin{array}{c}\text { SOF/RBV } 16 \text { week } \\
(\mathrm{n}=95)\end{array}$ \\
\hline Overall & $91 \%$ & $67 \%$ & $67 \%$ & $78 \%$ & $0 \%$ & $50 \%$ & $73 \%$ \\
\hline GT 2 & N/A & $97 \%$ & $78 \%$ & $93 \%$ & $0 \%$ & $86 \%$ & $94 \%$ \\
\hline GT 3 & N/A & $56 \%$ & $63 \%$ & $61 \%$ & $0 \%$ & $30 \%$ & $62 \%$ \\
\hline Noncirrhotic & $93 \%$ & $72 \%$ & $74 \%$ & $81 \%$ & $0 \%$ & $61 \%$ & $76 \%$ \\
\hline Cirrhotic & $80 \%$ & $47 \%$ & $38 \%$ & $61 \%$ & $0 \%$ & $31 \%$ & $66 \%$ \\
\hline
\end{tabular}

Table 2. SVR12 vs SVR24.

\section{SVR12}

SVR24

Treatment-naïve patients

GT 1, 4, 5, 6 overall

GT 1

GT 4

GT 5 and GT 6

$91 \%$

$90 \%$

$96 \%$

$100 \%$

$67 \%$

$78 \%$

$51 \%$

Previously treated (12 week regimen)

Previously treated (16 week regimen)

\author{
Treatment-naive \\ Interferon unable
}

Treatment-naive and experienced GT 2, 3 patients

Treatment-naive
Interferon unable
Previously treated (12 week regimen)
Previously treated (16 week regimen)

(1)

91\%

$90 \%$

$96 \%$

$100 \%$ 
The additional data back up the 12-week SVR. "In all the studies, the 24-week results are almost identical [to the 12-week SVR]. We detected durability of that response," said Dr. Kowdley. The studies also suggest that interferon is not needed to achieve SVR in genotypes 2 and 3, although Dr. Kowdley said that trials of interferon-sparing regimens are underway [10].

\section{Verification for Sofosbuvir}

Sofosbuvir, an orally administered nucleotide analogue inhibitor of the HCV NS5B polymerase, exerts potent antiviral activity against HCV genotypes 1 through 6 . This drug is meant to be taken once daily at a dose of 400 mg. Sofosbuvir has been extensively studied in various patient populations in combination with PEG-IFN/ribavirin, as well as with other direct-acting antiviral agents in treatment-naive patients with genotype $1 \mathrm{HCV}$ infection [11]-[15].

The FDA advisory committee reviewed primary efficacy and safety data from a series of clinical trials. The data supported the possibility of effectively treating HCV infection with a brief, well-tolerated, all-oral, oncedaily regimen that has no known safety issues and no resistance development. Phase 3 trials of sofosbuvir in treatment-naive patients with hepatitis $C$ virus genotypes 1 through 6 demonstrated that patients with genotype 1 infection have excellent treatment response that is superior overall to published response rates for combination therapy and currently available triple therapies. For patients with genotypes 2 and 3, efficacy was similar between an IFN-free sofosbuvir regimen and a standard PEG-IFN/ribavirin regimen [14] [15].

More evidence for Sofosbuvir

Numerous studies have emerged; a brief overview of a few representative studies of sofosbuvir in combination with other direct-acting antiviral agents is offered here:

- Sofosbuvir was combined with simeprevir with and without ribavirin; SVR rates of 93\% - 96\% were reported [14].

- Sofosbuvir plus the NS5A inhibitor daclatasvir led to SVR at 12 weeks (SVR12) rates of 86\% - 100\% [16].

- Ledipasvir is a novel HCV NS5A inhibitor that has shown potent antiviral activity against genotypes 1a and 1b HCV infection [17] [18]. It is active against HCV with the S282T mutation, the only variant known to reduce susceptibility to sofosbuvir [19]. All treatment-naive patients and prior null responders (noncirrhotic) who received 12 weeks of sofosbuvir and ledipasvir plus ribavirin achieved high SVR12 rates (95\% - 100\%). Patients treated for 12 weeks had a similar response to patients who received 8 weeks of therapy, suggesting that this shorter treatment strategy might be sufficient for noncirrhotic patients who have not previously been treated for HCV.

In all of these studies, sofosbuvir was well tolerated, with a low incidence of adverse events. In conjunction with the suggested brief duration of this regimen, this indicates that drug combinations should improve treatment adherence compared with IFN-based treatment. Traditional predictors of response, such as IL28B genotype and baseline viral load, do not seem to affect response rates. Other large multicenter trials are under way, designed to address the optimal treatment combination and duration, the need for ribavirin, and the efficacy in patients with compensated cirrhosis in both treatment-naive and previously treated patients [19].

\section{Data on the Drug}

Presentations offered at the AASLD's Liver Meeting 2013 abetted the data submitted to the FDA. Several studies reported pan-genotypic efficacy and safety of sofosbuvir, as well as other potential uses for this agent in various drug combinations and in various populations, including the following [2]:

In a phase 3 trial, 12 weeks of sofosbuvir plus ribavirin demonstrated high SVR rates in a predominantly treatment-experienced patient population with genotypes 2 and $3 \mathrm{HCV}$ infection, with higher response rates in patients infected with genotype 2 than in those infected with genotype 3 HCV [20].

- In a phase 2, randomized, open-label study, the combination of sofosbuvir plus simeprevir plus ribavirin for 12 or 24 weeks in patients with HCV genotype 1 infection resulted in high SVRs. This study included null responders and patients with cirrhosis [21].

- Sofosbuvir plus ledipasvir given in a fixed combination elicited a rapid decline in HCV RNA levels in all patient populations, with no viral breakthrough. In treatment-naive patients with genotype 1 infection and without cirrhosis, a reduction in duration of therapy from 12 to 6 weeks increased the rate of relapse [22]. In genotype 1-infected patients who were prior null responders and had cirrhosis, the addition of ribavirin to 
sofosbuvir and ledipasvir reduced the rate of relapse.

- Treatment-naive patients with HCV genotype 2 and 3 who were coinfected with HIV achieved high SVR12 rates with an IFN-free, oral regimen of sofosbuvir plus ribavirin [23]. The SVR12 rates were $76 \%$ among patients with HCV genotype 1, 88\% among those with genotype 2, and 67\% among those with genotype 3; these are similar to the rates observed for patients infected with HCV only. These preliminary data suggest that sofosbuvir plus ribavirin treatment was well tolerated and safe, even with the coadministration of multiple antiretroviral drugs.

A Markov model, developed to evaluate the long-term outcomes of sofosbuvir-based therapy for HCV infection, indicated that regimens incorporating this agent are highly effective in preventing progression to advanced liver disease [24].

\section{New Opportunities Bring New Challenges}

Recurrence of HCV infection is the most common cause of graft loss and mortality in HCV-infected liver transplant recipients. IFN-based post-transplantation antiviral regimens, including those using protease inhibitors, are poorly tolerated and achieve SVRs that are lower than those in nontransplant patients.

Administration of sofosbuvir plus ribavirin after liver transplantation in the setting of established HCV recurrence was well tolerated, and approximately $80 \%$ of patients achieved an early SVR at 4 weeks. There were no episodes of rejection or drug interaction, and there was no apparent effect of sofosbuvir on serum levels of immunosuppressive medications, offering the potential for an all-oral therapy for treatment of HCV infection after liver transplantation [25]. Sofosbuvir and ribavirin may, in fact, be used in the pretransplant phase to prevent recurrence of HCV infection after transplantation [26].

The availability of these agents will provide unprecedented opportunities for off-label use of these therapies in many patients, including those with decompensated cirrhosis or chronic kidney disease, pediatric populations, and those with HIV coinfection. Because many of these populations represent relatively small numbers of patients with $\mathrm{HCV}$, it may be difficult to accumulate the requisite data and possibly cost-prohibitive for manufacturers to apply for FDA approval [27].

The bottom line is that simpler, shorter, and safer strategies for treatment of patients infected with HCV are at hand.

\section{Conclusion}

At end, 2 novel direct-acting antiviral agents—sofosbuvir and simeprevir-target various components of the HCV genome. Advantages of these drugs include a high barrier to viral resistance, a shorter duration of treatment, once-daily dosing, absence of food restrictions, few clinically significant drug interactions, and similar efficacy in all genotypes. This will offer clinicians new options as well as new challenges. A recent review addressed some of these anticipated issues, such as the off-label use of HCV medications and the roles of the FDA, consumer pressure, medical society guidelines, and third-party payers [27].

\section{References}

[1] Page, K., Morris, M.D., Hahn, J.A., Maher, L. and Prins, M. (2013) Injection Drug Use and Hepatitis C Virus Infection in Young Adult Injectors: Using Evidence to Inform Comprehensive Prevention. Clinical Infectious Diseases, 45, S32S38.

[2] Ghany, M.G., Nelson, D.R., Strader, D.B., Thomas, D.L. and Seeff, L.B. (2011) American Association for the Study of Liver Diseases. An Update on Treatment of Genotype 1 Chronic Hepatitis C Virus Infection: 2011 Practice Guideline by the American Association for the Study of Liver Diseases. Hepatology, 54, 1433-1444.

http://dx.doi.org/10.1002/hep.24641

http://www.aasld.org/practiceguidelines/Documents/AASLDUpdateTreatmentGenotype1HCV11113.pdf

[3] Hézode, C., Fontaine, H., Dorival, C., et al. (2013) CUPIC Study Group. Triple Therapy in Treatment-Experienced Patients with HCV-Cirrhosis in a Multicentre Cohort of the French Early Access Programme (ANRS CO20-CUPIC)NCT01514890. Journal of Hepatology, 49, 434-441. http://dx.doi.org/10.1016/j.jhep.2013.04.035

[4] Sulkowski, M.S., Cooper, C., Hunyady, B., et al. (2011) Management of Adverse Effects of Peg-IFN and Ribavirin Therapy for Hepatitis C. Nature Reviews Gastroenterology \& Hepatology, 8, 212-223.

http://dx.doi.org/10.1038/nrgastro.2011.21 
[5] Heim, M.H. (2013) 25 Years of Interferon-Based Treatment of Chronic Hepatitis C: An Epoch Coming to an End. Nature Reviews Immunology, 13, 535-342. http://dx.doi.org/10.1038/nri3463

[6] Lewis, R. (2013) Hepatitis C: 6 Prevention Strategies That Work. http://www.medscape.com/viewarticle/808539

[7] Ghany, M.G., Strader, D.B., Thomas, D.L. and Seeff, L.B. (2009) Diagnosis, Management, and Treatment of Hepatitis C: An Update. Hepatology, 46, 1335-1374. http://dx.doi.org/10.1002/hep.22759

[8] Greg Fitz, M.D. (2013) Promising New Era in Hepatitis C Treatment. The Liver Meeting: American Association for the Study of Liver Diseases (AASLD), Washington DC, 11 November 2013.

[9] Lawitz, E., Lalezari, J.P. and Hassanein, T. (2013) Sofosbuvir in Combination with Peginterferon Alfa-2a and Ribavirin for Non-Cirrhotic, Treatment-Naive Patients with Genotypes 1, 2, and 3 Hepatitis C Infection: A Randomised, Double-Blind, Phase 2 Trial. The Lancet Infectious Diseases, 13, 401-408. http://dx.doi.org/10.1016/S1473-3099(13)70033-1

[10] Liver Center of Excellence at the Digestive Disease; Institute at the Virginia Mason Medical Center in Seattle, Washington (2013) Hepatitis C Drug Sofosbuvir Still Effective at 24 Weeks. American College of Gastroenterology (ACG) 2013 Annual Scientific Meeting and Postgraduate Course, San Diego, 11-16 October 2013, Abstract 38.

[11] Kowdley, K.V., Lawitz, E., Crespo, I., et al. (2013) Sofosbuvir with Pegylated Interferon Alfa-2a and Ribavirin for Treatment-Naive Patients with Hepatitis C Genotype-1 Infection (ATOMIC): An Open-Label, Randomised, Multicentre Phase 2 Trial. Lancet, 381, 2100-2107.

[12] Kowdley, K.V., Lawitz, E., Crespo, I., et al. (2013) Sofosbuvir with Pegylated Interferon Alfa-2a and Ribavirin for Treatment-Naive Patients with Hepatitis C Genotype-1 Infection (ATOMIC): An Open-Label, Randomised, Multicentre Phase 2 Trial. Lancet, 381, 2100-2107. http://dx.doi.org/10.1016/S0140-6736(13)60247-0

[13] Lawitz, E., Mangia, A., Wyles, D., et al. (2013) Sofosbuvir for Previously Untreated Chronic Hepatitis C Infection. The New England Journal of Medicine, 368, 1878-1887. http://dx.doi.org/10.1056/NEJMoa1214853

[14] Lawitz, E., Ghalib, R., Rodriguez-Torres, M., et al. (2013) COSMOS Study: SVR4 Results of a Once Daily Regimen of Simeprevir (TMC435) plus Sofosbuvir (GS-7977) with or without Ribavirin in HCV Genotype 1 Null Responders. Program and Abstracts of the 20th Conference on Retroviruses and Opportunistic Infections, Atlanta, 3-6 March 2013, Abstract 155LB.

[15] Jacobson, I.M., Gordon, S.C., Kowdley, K.V., et al. (2013) POSITRON Study; FUSION Study. Sofosbuvir for Hepatitis C Genotype 2 or 3 in Patients without Treatment Options. The New England Journal of Medicine, 368, 1867-1877. http://dx.doi.org/10.1056/NEJMoa1214854

[16] Poordad, F., Lawitz, E., Kowdley, K.V., et al. (2013) Exploratory Study of Oral Combination Antiviral Therapy for Hepatitis C. The New England Journal of Medicine, 386, 45-53. http://dx.doi.org/10.1056/NEJMoa1208809

[17] Lawitz, E., Poordad, F., Pang, P.S., et al. (2013) Sofosbuvir and Ledipasvir Fixed-Dose Combination with and without Ribavirin in Treatment-Naive and Previously Treated Patients with Genotype 1 Hepatitis C Virus Infection (LONESTAR): An Open-Label, Randomised, Phase 2 Trial. The Lancet, 383, 515-523.

[18] Lawitz, E.J., Gruener, D., Hill, J.M., et al. (2012) A Phase 1, Randomized, Placebo-Controlled, 3-Day, Dose-Ranging Study of GS-5885, an NS5A Inhibitor, in Patients with Genotype 1 Hepatitis C. Journal of Hepatology, 57, 24-31.

[19] Cheng, G., Peng, B., Corsa, A., et al. (2012) Antiviral Activity and Resistance Profile of the Novel HCV NS5A Inhibitor GS-5885. Journal of Hepatology, 56, S464.

[20] Zeuzem, S., Dusheiko, G.M., Salupere, R., et al. (2013) Sofosbuvir + Ribavirin for 12 or 24 Weeks for Patients with HCV Genotype 2 or 3: The VALENCE Trial. Program and abstracts of the 64th Annual Meeting of the American Association for the Study of Liver Diseases, Washington DC, 1-5 November 2013, Abstract 1085.

[21] Jacobson, I., Ghalib, R.H., Rodriguez-Torres, M., et al. (2013) SVR Results of a Once-Daily Regimen of Simeprevir (TMC435) plus Sofosbuvir (GS-7977) with or without Ribavirin in Cirrhotic and Non-Cirrhotic HCV Genotype 1 Treatment-Naïve and Prior Null Responder Patients: The COSMOS Study. Program and Abstracts of the 64th Annual Meeting of the American Association for the Study of Liver Diseases, Washington DC, 1-5 November 2013, Abstract LB-3.

[22] Gane, E.J., Stedman, C.A., Hyland, R.H., et al. (2013) Once Daily Sofosbuvir/Ledipasvir Fixed Dose Combination with or without Ribavirin: The ELECTRON Trial. Program and Abstracts of the 64th Annual Meeting of the American Association for the Study of Liver Diseases, Washington DC, 1-5 November 2013, Abstract 21.

[23] Sulkowski, M.S., Rodriguez-Torres, M., Lalezari, J.P., et al. (2013) All-Oral Therapy with Sofosbuvir plus Ribavirin for the Treatment of HCV Genotype 1, 2, and 3 Infection in Patients Co-Infected with HIV (PHOTON-1). Program and Abstracts of the 64th Annual Meeting of the American Association for the Study of Liver Diseases, Washington DC, 1-5 November 2013, Abstract 212.

[24] Cure, S., Guerra, I. and Dusheiko, G. (2013) Long-Term Outcomes of Sofosbuvir (SOF) for the Treatment of Chronic Hepatitis C Infected (CHC) Patients. Program and Abstracts of the 64th Annual Meeting of the American Association 
for the Study of Liver Diseases, Washington DC, 1-5 November 2013, Abstract 1107.

[25] Charlton, M.R., Gane, E.J., Manns, M.P., et al. (2013) Sofosbuvir and Ribavirin for the Treatment of Established Recurrent Hepatitis C Infection after Liver Transplantation: Preliminary Results of a Prospective, Multicenter Study. Program and Abstracts of the 64th Annual Meeting of the American Association for the Study of Liver Diseases, Washington DC, 1-5 November 2013, Abstract LB-2.

[26] Curry, R., Forns, X., Chung, R.T., et al. (2013) Pretransplant Sofosbuvir and Ribavirin to Prevent Recurrence of HCV Infection after Liver Transplantation. Program and Abstracts of the 64th Annual Meeting of the American Association for the Study of Liver Diseases, Washington DC, 1-5 November 2013, Abstract 213.

[27] Aronsohn, A., Reau, N. and Jensen, D. (in Press) Preparing for the Uncertain yet Inevitable: Off Label Combinations of Antiviral Agents in HCV. Hepatology. 
Scientific Research Publishing (SCIRP) is one of the largest Open Access journal publishers. It is currently publishing more than 200 open access, online, peer-reviewed journals covering a wide range of academic disciplines. SCIRP serves the worldwide academic communities and contributes to the progress and application of science with its publication.

Other selected journals from SCIRP are listed as below. Submit your manuscript to us via either submit@scirp.org or Online Submission Portal.
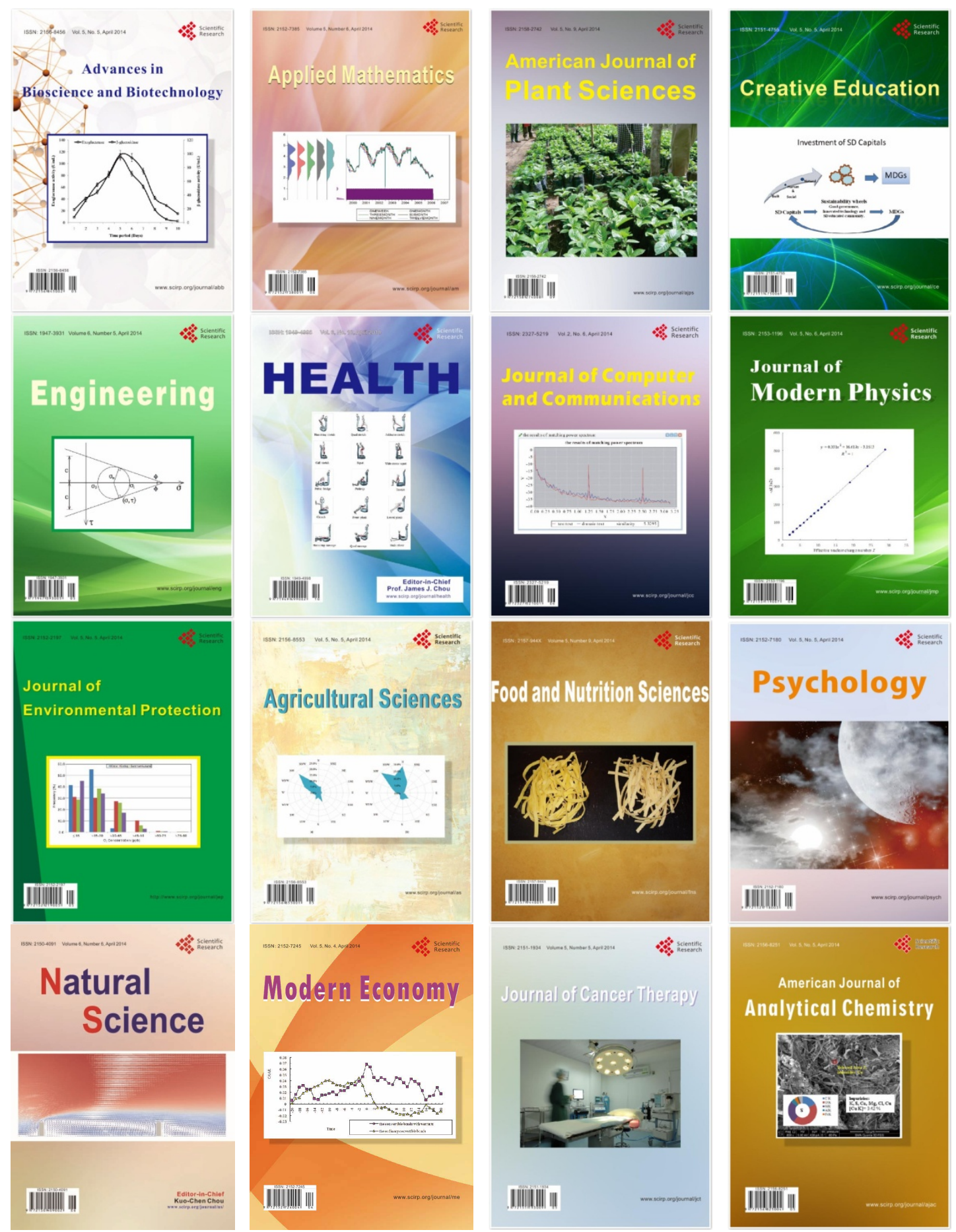